\title{
Perfil materno de gravidez na adolescência: dados preliminares do ano de 2021 no
}

\section{estado do Paraná}

\author{
Maternal pregnancy profile in adolescence: preliminary data for the year 2021 in the state of \\ Paraná
}

Perfil de embarazo materno en adolescencia: datos preliminares para el año 2021 en el estado de

Paraná

Recebido: 30/09/2021 | Revisado: 09/10/2021 | Aceito: 13/10/2021 | Publicado: 16/10/2021

\author{
Renan Martins Maciel \\ ORCID: https://orcid.org/0000-0002-7398-7024 \\ Faculdade Cristo Rei, Brasil \\ E-mail: renanmmaciel@hotmail.com \\ Tatiane Renata Fagundes \\ ORCID: https://orcid.org/0000-0003-4634-360X \\ Faculdade Cristo Rei, Brasil \\ E-mail: tatiane@faccrei.edu.br
}

\begin{abstract}
Resumo
O presente artigo apresenta um estudo ecológico do perfil materno e acompanhamento da gravidez na adolescência, através de dados preliminares do ano de 2021 no estão do Paraná e a atuação do profissional de enfermagem nessa situação. A pesquisa tem como objetivo avaliar os parâmetros sociais, econômicos e familiares na gestação de adolescentes até 19 anos de idade, verificando a possível relação com a prematuridade, baixo peso ao nascer e outro parâmetros de saúde materno fetal. Das gestações ocorridas no Paraná até o mês de agosto de 2021, 11,23\% ocorreram em adolescentes solteiras de 10 a 19 anos de idade, com predominância nos 15 a 19 anos. Esse fato torna se importante para a saúde pública e sociedade, pois uma gravidez nessa idade aumenta a evasão escolar, maiores chances de complicações materno-fetais, e pode causar conflitos familiares. Nesse sentido o enfermeiro tem o papel de prevenção e orientação direta aos adolescentes de forma apropriada a sua idade e capacidade de compreensão, de acolhimento a adolescente grávida, e de promoção da saúde da mãe e do recém-nascido após o nascimento.
\end{abstract}

Palavras-chave: Gravidez; Adolescência; Enfermeiro; Recém-nascido.

\begin{abstract}
This article presents an ecological study of the maternal profile and monitoring of teenage pregnancy, through preliminary data from the year 2021 in Paraná State and the role of the nursing professional in this situation. The research aims to evaluate the social, economic and family parameters in the pregnancy of adolescents up to 19 years of age, verifying the possible relationship with prematurity, low birth weight and other maternal-fetal health parameters. Of the pregnancies that occurred in Paraná until the month of August 2021, 11.23\% occurred in single adolescents aged 10 to 19 years old, with a predominance between 15 and 19 years old. This fact becomes importante or public health and society, as a pregnancy at this age increases school dropout, greater chances of maternal-fetal complications, and can cause family conflicts. In this sense, the nurse has the role of prevention and direct guidance to adolescents in a manner appropriate to their age and ability to understand, welcoming the pregnant adolescent, and promoting the health of the mother and newborn after birth.
\end{abstract}

Keywords: Pregnancy; Adolescence; Nurse; Newborn.

\section{Resumen}

Este artículo presenta un estudio ecológico del perfil materno y el seguimiento del embarazo adolescente, através de datos preliminares del año 2021 en el estado de Paraná y el rol del profesional de enfermería en esta situación. La investigación tiene como objetivo evaluar los parámetros sociales, económicos y familiares en el embarazo de adolescentes hasta los 19 años, verificando la posible relación con la prematuridad, el bajo peso al nacer y otros parámetros de salud materno-fetal. De los embarazos ocurridos en Paraná hasta el mes de agosto de 2021, el 11,23\% ocurrió en adolescentes solteras de 10 a 19 años, con predominio entre 15 y 19 años. Este hecho se vuelve importante para la salud pública y la sociedad, ya que un embarazo a esta edad aumenta la deserción escolar, aumenta las posibilidades de complicaciones materno-fetales y puede generar conflictos familiares. En este sentido, la enfermera tiene el rol de prevención y orientación directa a los adolescentes de manera adecuada a su edad y capacidad de 
comprensión, acogiendo a la adolescente embarazada y promoviendo la salud de la madre y el recién nacido después del nacimiento.

Palabras clave: Embarazo; Adolescencia; Enfermera; Recién Nacido.

\section{Introdução}

A adolescência é definida como a fase de transição do ser humano entre a dependência infantil e a emancipação do jovem adulto, podendo ser determinado dos pontos de vista sociológico, psicanalítico e o biológico, entre os 10 a 19 anos incompletos (Baldoino et al., 2018; Nasio \& tradução André Telles, 2011).

Essa fase é marcada por tomada de decisões que impactam todo o restante da vida, associada à noção de crise, desordem, que por vezes geram problemas sociais associados a riscos, como gravidez indesejada, infecções sexualmente transmissíveis, uso de drogas ilícitas e até mesmo morte frente à violência.

Segundo o Departamento de Informática do Sistema Único de Saúde (DATASUS), através do Sistema Nacional de Nascidos Vivos (SINASC), a gravidez na adolescência nos últimos 20 anos no Brasil chegou a 37,2\% do total de gestações, e no ano de 2020, a cada mil brasileiras entre 15 a 19 anos, 53 adolescentes tornaram-se mãe, o que gera um grande impacto no futuro das jovens (Brasil, 2021).

Segundo a Organização Mundial da Saúde (OMS), a mortalidade dos filhos dessas adolescentes é alta no primeiro ano de vida, devido aos fatores de risco desenvolvidos durante uma gestação nessa fase da vida (E. F. V. de Oliveira et al., 2010).

Alguns estudos mostram que as consequências na vida da mãe adolescente refletem na vida do neonato; foi observado neonatos com baixo peso, sofrimento fetal, rompimento de membranas, prematuridade, maior frequência de apgar mais baixo, doenças respiratórias, trauma obstétrico. Um estudo conduzido no Brasil mostrou que a maioria dos bebês que necessitaram de cuidados intensivos (UTI) após o nascimento, eram de mulheres com idade entre 10 a 19 anos quando comparado às adultas (Organización Panamericana de la Salud \& Organización Mundial de la Salud, 2016; Valentim, 2018).

A gravidez na adolescência representa um grave problema de saúde pública, envolvendo mudanças não somente físicas, mas também psicológicas e socioeconômicas. Uma gestação nessa idade está relacionada a baixa autoestima, desvalorização perante a sociedade e família, baixas expectativas futuras, situações de estresse, sofrimento, que podem culminar em exposição maior a álcool e drogas, além de assistência pré-natal inadequada, que promoverá prejuízos na saúde materna e neonatal (Barreto et al., 2011; Duarte et al., 2018; Lima et al., 2017; Organización Panamericana de la Salud \& Organización Mundial de la Salud, 2016; Schwanke \& Pinto, 2011).

As causas associadas a gestação na adolescência remota de situações determinantes sociais, como à falta de orientação, baixa renda, onde grande parcela da população vive em situação de vulnerabilidade, deste modo acarretando maior densidade domiciliar; baixa escolaridade, risco de violência, aumento do desamparo econômico e menor índice de desenvolvimento humano (P. R. de Oliveira et al., 2018). Até mesmo a falta de comunicação mais aberta entre pais filhos pode ser um fator influente (Minas Gerais, 2006). Os problemas socioeconômicos, de saúde e familiares desencadeados começam desde a descoberta da gestação pela família, gerando conflitos devido a crenças, valores e atitudes, associados a despesas financeiras que aumentam, e a mudanças na vida da adolescente (Piccinini et al., 2004).

O objetivo deste artigo é avaliar os parâmetros sociais, econômicos e familiares na gestação de adolescentes até 19 anos de idade no estado do Paraná, através de dados preliminares do ano de 2021 e verificar a possível relação com a prematuridade, baixo peso ao nascer e outro parâmetros de saúde materno fetal, e destacar a atuação da enfermagem na prevenção da gravidez na adolescência. 


\section{Metodologia}

Este é um estudo ecológico utilizando informações das Declarações de Nascidos Vivos dos RN de mães adolescentes (10-19 anos), através do DATASUS, de ocorrência no estado do Paraná, correspondente ao ano de 2021, até mês de agosto.

As variáveis utilizadas foram: perfil materno, faixa etária da mãe (categorizada até 14 anos, ou 15 a 19 anos de idade), estado civil, escolaridade; perfil de gestação e parto: número de consultas pré-natal, inicio do pré-natal, tipo de parto (normal ou cesariano), peso ao nascer e idade gestacional.

Segundo a OMS (WHO, 2019) os neonatos são classificados de baixo peso $(<2500 \mathrm{~g})$, de peso insuficiente (2500$2999 \mathrm{~g}$ ) e peso adequado (> 3000g); e prematuros quando nascidos com menos de 37 semanas de gestação. Como preconizado pelo Ministério da Saúde o pré-natal é considerado incompleto com menos de 6 consultas e completo com 6 consultas ou mais, uma vez que, o Programa de Humanização no Pré-Natal e Nascimento (PHPN) estabelece o número mínimo de 6 consultas (Brasil, 2000).

Os dados foram analisados através de uma estatística descritiva. Por se tratar de uma pesquisa envolvendo apenas dados de domínio público, sem envolvimento de seres humanos, a pesquisa é dispensada da avaliação pelo Comitê de Ética em Pesquisa.

\section{Resultados e Discussão}

Entre os anos de 2012 a agosto de 2021, ocorreram 1.443.648 gestações no estado do Paraná (Gráfico 1). Desse total 220.744 correspondem a gestações em adolescentes $(15,29 \%)$.

Gráfico 1: Total de gestações no estado do Paraná, entre os anos de 2012 a agosto de 2021.

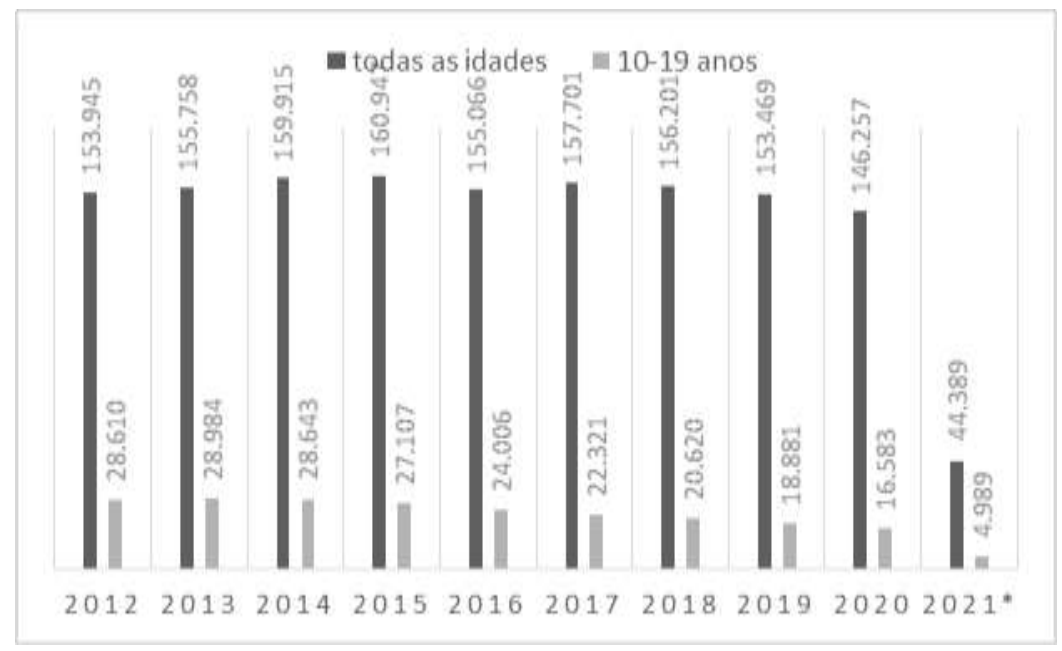

Fonte: Elaborado pelos autores segundo dados do MS - Sistema de Informações sobre Nascidos Vivos - SINASC.

Em janeiro de 2019 a Lei 13.798 foi incluída no Estatuto da Criança e do Adolescente (Lei 8.069/90), instituindo a semana nacional de prevenção da gravidez na adolescência, a ser realizada anualmente, a partir de $1^{\circ}$ de fevereiro de 2019 , com objetivo de disseminar as informações sobre medidas preventivas e educativas que contribuíam para a redução da incidência de gravidez na adolescência (OMS, 2019). De fato, observa-se uma queda no número de casos de gestações em adolescentes de 10 a 19 anos, de forma gradativa.

A seguir analisamos alguns dados relacionados as características sociais das gestantes com idades entre 10 e 19 anos, durante o ano de 2021, através de dados disponíveis no SINASC até o momento (Tabela 1). Nesse ano ocorreram 4.989 
gestações em adolescentes de 10 a 19 anos no estado do Paraná, 96\% entre as idades de 15 e 19 anos, número que corresponde a 11,23\% de todas as gestações ocorridas no estado até agosto de 2021 (Tabela 1).

Quanto ao estado civil, 70,01\% delas estavam solteiras nesse momento, e 21,42\% em união estável. É visível que número de mães adolescentes solteiras é muito maior, muitas são vítimas de gravidez acidental, por abuso sexual, falta de orientação sobre os métodos contraceptivos. A maioria das gestantes adolescentes $(77,49 \%)$ ainda estava cursando ensino médio, ou havia concluído a pouco tempo no momento da gestação (Tabela 1).

Sobre esses dados, as adolescentes grávidas solteiras, devido sua pouca idade não apresentam uma relação afetiva estável com o parceiro, e na maioria das vezes a gravidez não foi planejada. Esses fatores geram pensamentos e atitudes contraditórias, especialmente em largar os estudos e ter que trabalhar, o que se agrava quando não existe apoio da família, além das dificuldades psicológicas e sociais de uma gravidez na adolescência (Bocardi \& Cano, 1998).

Tabela 1. Números absolutos e porcentagem do perfil materno de gestantes adolescentes, SINASC, 2021, Paraná, Brasil.

\begin{tabular}{|c|c|c|c|}
\hline Características & Variáveis & $\begin{array}{c}n \\
\text { (n total de gestantes: } 4989)\end{array}$ & $\%$ \\
\hline \multirow{2}{*}{ Idade materna (anos) } & 0 a 14 anos & 192 & $3,85 \%$ \\
\hline & 15 a 19 anos & 4.797 & $96,15 \%$ \\
\hline \multirow{6}{*}{ Escolaridade } & 1 a 3 anos & 46 & $0,92 \%$ \\
\hline & 4 a 7 anos & 939 & $18,82 \%$ \\
\hline & 8 a 11 anos & 3.836 & $76,88 \%$ \\
\hline & Mais de 12 anos & 135 & $2,70 \%$ \\
\hline & Nenhuma & 1 & $0,02 \%$ \\
\hline & Não informado & 32 & $0,64 \%$ \\
\hline \multirow{6}{*}{ Estado civil } & Solteira & 3493 & $70,01 \%$ \\
\hline & Casada & 374 & $7,49 \%$ \\
\hline & Viúva & 1 & $0,02 \%$ \\
\hline & Separada judicialmente/Divorciada & 8 & $0,16 \%$ \\
\hline & União estável & 1069 & $21,42 \%$ \\
\hline & Branco/Ignorado & 44 & $0,88 \%$ \\
\hline
\end{tabular}

Fonte: Elaborado pelos autores segundo dados do MS - Sistema de Informações sobre Nascidos Vivos - SINASC.

Na América Latina, o Brasil tem uma das maiores taxas de gestação na adolescência, são dados consideráveis e precisam de planejamento e ações com urgência (Sociedade Brasileira de Pediatria, 2019), pois a gestação nessa faixa etária está associada a uma série de agravos, tanto socioeconômicos, como nas relações familiares e pessoais, e um elevado risco de óbito da mãe e do bebê, ocasionando aborto espontâneo, prematuridade, eclampsia, anemia, depressão pós-parto, entre outros (Lima et al., 2017).

A Tabela 2 apresenta os dados referentes ao perfil de gestação, pré-natal e de nascidos vivos de mães adolescentes, no ano de 2021, no estado do Paraná. 
Tabela 2. Números absolutos e porcentagem do perfil da gestação, parto de gestantes adolescentes e recém-nascidos, SINASC, 2021, Paraná, Brasil.

\begin{tabular}{|c|c|c|c|}
\hline Características & Variáveis & $\begin{array}{c}n \\
\text { (n total de gestantes: } 4989)\end{array}$ & $\%$ \\
\hline \multirow{10}{*}{$\begin{array}{c}\text { Mês de gestação de início de } \\
\text { pré-natal }\end{array}$} & $1^{\circ}$ mês & 1105 & $22,14 \%$ \\
\hline & $2^{\circ}$ mês & 2030 & $40,68 \%$ \\
\hline & $3^{\circ}$ mês & 913 & $18,30 \%$ \\
\hline & $4^{\circ}$ mês & 367 & $7,35 \%$ \\
\hline & $5^{\circ}$ mês & 199 & $3,98 \%$ \\
\hline & $6^{\circ}$ mês & 120 & $2,40 \%$ \\
\hline & $7^{\circ}$ mês & 91 & $1,82 \%$ \\
\hline & $8^{\circ}$ mês & 33 & $0,66 \%$ \\
\hline & $9^{\circ}$ mês & 9 & $0,180 \%$ \\
\hline & Branco/Ignorado & 122 & $2,44 \%$ \\
\hline \multirow{9}{*}{ Idade gestacional } & $\begin{array}{l}\text { Menos de } 22 \text { semanas } \\
\text { (prematuros extremos) }\end{array}$ & 3 & $0,060 \%$ \\
\hline & 22 a 27 semanas & 37 & $0,74 \%$ \\
\hline & 28 a 31 semanas & 62 & $1,24 \%$ \\
\hline & 32 a 36 semanas & 470 & $9,42 \%$ \\
\hline & $\begin{array}{l}\text { Menos de } 37 \text { semanas } \\
\text { (prematuros) }\end{array}$ & 577 & $11,56 \%$ \\
\hline & 37 a 41 semanas & 2.570 & $51,51 \%$ \\
\hline & Mais de 38 semanas (a termo) & 1.134 & $22,73 \%$ \\
\hline & 42 semanas e mais & 81 & $1,62 \%$ \\
\hline & Branco/Ignorado & 50 & $1,00 \%$ \\
\hline \multirow{5}{*}{ Consultas pré-natal } & Nenhuma & 54 & $1,08 \%$ \\
\hline & De 1 a 3 & 234 & $4,69 \%$ \\
\hline & De 4 a 6 & 843 & $16,89 \%$ \\
\hline & De 7 a mais & 3848 & $77,12 \%$ \\
\hline & Branco/ Ignorado & 10 & $0,20 \%$ \\
\hline \multirow{4}{*}{ Peso (g) ao nascer } & $<1500$ & 65 & $1,30 \%$ \\
\hline & 1500 a 2499 & 414 & $8,29 \%$ \\
\hline & 2500 a mais & 4510 & $90,39 \%$ \\
\hline & Branco/Ignorado & 0 & - \\
\hline \multirow{3}{*}{ Tipo de Parto } & Vaginal & 2621 & $52,53 \%$ \\
\hline & Cesáreo & 2360 & $47,30 \%$ \\
\hline & Branco/Ignorado & 8 & $0,16 \%$ \\
\hline
\end{tabular}

Fonte: Elaborado pelos autores segundo dados do MS - Sistema de Informações sobre Nascidos Vivos - SINASC. 
O Programa de Humanização no Pré-Natal e Nascimento (PHPN) do Ministério da Saúde (MS), criado em junho de 2000, busca a redução das altas taxas de morbidade e mortalidade materna e perinatal, no sentido de assegurar o acesso, e melhorar a cobertura e qualidade do acompanhamento pré-natal, da assistência ao parto, puerpério e neonatal. Esse programa preconiza que durante o acompanhamento pré-natal, a gestante deve passar por no mínimo 6 consultas, considerando ainda como incompleto o acompanhamento pré-natal com 6 consultas (Brasil, 2000). É ainda recomendável que o pré-natal se inicie com a descoberta da gravidez, sendo extremamente importante para a saúde física e psicológica da mãe e do feto, devendo ocorrer pelo menos uma consulta no primeiro trimestre de gravidez, duas no segundo, três no terceiro, entre a $34^{\mathrm{a}}$ e $38^{\mathrm{a}}$ semana é indicado uma consulta a cada duas semanas, e após a $38^{\mathrm{a}}$ semanas devem ser realizadas consultas semanais até o parto (Brasil, 2000).

De acordo com os dados encontrados no SINASC, para o ano de 2021, 77,12\% das gestantes adolescentes realizaram 7 ou mais consultas pré-natal, e 16,89\% foram de 4 a 6 consultas pré-natal, número considerado incompleto. Uma possibilidade para esse resultado é a questão de que $18,30 \%$ delas descobriram e começaram esse acompanhamento no $3^{\circ}$ mês de gestação.

Nenhuma consulta pré-natal foi registrada em 1,08\% das gestantes, o que pode ocorrer por não saberem a importância do pré-natal, ou até mesmo o desejo de esconder sua gravidez, ou desconhecimento da gestação (Lansky et al., 2014).

A idade gestacional menor que 37 semanas é considerada prematura, e em nossa pesquisa 11,56\% das gestações apresentaram esse número. Maior parte dos dados encontrados (51,51\%) para esse ano apontam que a idade gestacional foi de 37 a 41 semanas. O parto prematuro acontece quando o bebê nasce antes das 37 semanas de gestação, e pode ser desencadeado por vários fatores, entre eles a ruptura prematura da bolsa amniótica, anemia ou pré-eclâmpsia da gestante, e se diagnosticado como parto prematuro é necessário um acompanhamento hospitalar com urgência para o acompanhamento da mãe e do neonato (Oliveira et al., 2006).

Segundo a OMS, os recém-nascidos com peso menor que 2,500 gramas são considerados de baixo peso ao nascer, 2,500 a 2,999 gramas o peso é insuficiente, peso adequado quando apresentarem 3,000 gramas ou mais (WHO, 2019). Os dados mostram que $90,39 \%$ dos neonatos tinham ao nascer 2,500 gramas ou mais, $8,29 \%$ tinham peso insuficiente e $1,30 \%$ eram de baixo peso ao nascer.

O baixo peso ao nascimento do bebê, o que está diretamente relacionado as condições de nutrição da gestante e do bebê, e pode ocorrer devido à baixa ingestão alimentar ou falta de orientação durante o período pré-natal, associado a prematuridade, e caracteriza um grande fator de mortalidade neonatal, índice elevado em grávidas adolescentes (Suzuki et al., 2007).

A hora do parto da gestante adolescente é muito delicado e requer uma atenção muito grande, e em algumas situações pode ser decisivo por conta dos riscos de pré-eclâmpsia, eclampsia, anemia, asfixia na hora do parto, baixo peso ao nascer, prematuridade e desnutrição (J. C. Oliveira et al., 2006). Quanto ao tipo de parto, 52,53\% das gestantes tiveram do tipo vaginal, e 47,30\% parto cesáreo. O parto vaginal também conhecido como parto natural ocorre quase sem intervenções médicas, ou apenas se necessário, e o parto cirúrgico conhecido como cesárea, é realizado em hospitais com intervenção médica e anestesia (Oliveira et al., 2016).

Não observamos números que de fato seriam significativos da relação de prematuridade, baixo peso ao nascer com a gravidez na adolescência, porem destacamos que esse é um estudo preliminar do ano de 2021, o que pode mudar com as estatísticas futuras, no sentido de que a amostra observada ainda é pequena. Acreditamos também que muitos dados estão indisponíveis no SINASC, o que dificulta observarmos a real situação da gestação nessa faixa etária.

Na questão delicada da gravidez na adolescência o profissional de enfermagem tem uma atuação essencial em atender com uma abordagem de humanização da assistência, atendimento de qualidade, para que a mão adolescente se sinta acolhida, 
sendo necessário conhecer os direitos das políticas públicas que viabilizam a implementação de ações voltadas a esse assunto (Santos et al., 2008).

O enfermeiro pode desenvolver habilidades preventivas e educativas com os adolescentes da comunidade, planejando estratégias para prevenção da gravidez na adolescência, projetando ideias de promoção de saúde e prevenção de infecção sexualmente transmissíveis (ISTs) e de gravidez precoce, objetivando orientar e conscientizar sobre a inclusão de jovens a participar de ações de educação em saúde, com finalidade dos próprios adolescentes a tomarem suas decisões, e desenvolver a capacidade do seu autocuidado e prevenção (Ribeiro et al., 2016).

Entre tantas atividades, a enfermagem e a equipe multidisciplinar podem orientar na prevenção da gravidez na adolescência, formando vínculos para assegurar acesso a métodos anticoncepcionais e informações sobre educação sexual, retardando o início da vida sexual.

O uso de preservativos entre os jovens de 15 a 19 frequentemente não se dá de forma consistente em todas as relações (Ribeiro et al., 2016). Nesse sentido, a falta de informação adequada sobre o assunto pode ser resolvida com a educação sexual, que tem suma importância para evitar gravidez nessa faixa etária, pois só através disso os jovens desenvolveram um comportamento sexual responsável, respeitando o próximo, a igualdade e equidade de gênero, protegendo contra gravidez indesejada e prevenindo ISTs.

Um acolhimento ético a gestante adolescente, promovendo um ambiente favorável e seguro, ações intersetoriais e interdisciplinares de educação sexual para a prevenção da gravidez precoce, integração da família, escola e comunidade são papéis essenciais para a atuação do enfermeiro frente a esse problema de saúde pública (Glêdes et al., 2010).

A gravidez na adolescência precisa ser tratada de forma mais consistente principalmente na família, escola, e em todo âmbito social, com isso o enfermeiro está pronto para exercer essa função na área da saúde pode contribuir na prevenção desta gravidez.

A realidade dos adolescentes vem colocando desafios novos, e cabe aos profissionais de saúde orientar no campo de educação em saúde.

\section{Conclusão}

A gravidez na adolescência ainda acontece por conta do início precoce na vida sexual, falta de uso dos métodos contraceptivos, ou uso inadequado, e até mesmo por falta de orientação, já que o assunto sexo é tabu em muitas famílias e escolas.

A atuação do enfermeiro em relação a gravidez na adolescência, está ligada diretamente em demostrar como é o mundo exterior, visando sempre na implementação de novas propostas de intervenção na prevenção, promoção saúde e qualidade vida e esses jovens.

A gestação é um momento muito especial para uma mulher, mas requer muitos cuidados para que a gravidez ocorra de forma tranquila, realização do pré-natal desde o processo inicial até o dia do parto, ter a alimentação equilibrada e saudável para evitar o baixo peso do bebê, realização de atividades físicas para evitar estresse físico e emocional, e outros cuidados para que mãe e recém-nascido passem por esse processo da melhor forma possível.

\section{Referências}

Baldoino, L. S., Maria, S., Silva, N., Maria, A., Ribeiro, N., Kassyanne, E., \& Ribeiro, C. (2018). Educação em saúde para adolescentes no contexto escolar: relato de experiências. Rev Enferm UFPE on Line, 12(4), 1167. https://doi.org/10.5205/1981-8963-v12i4a230656p1161-1167-2018

Barreto, M. M. M., Gomes, A. M. T., Oliveira, D. C. de, Marques, S. C., \& Peres, E. M. (2011). Representação social da gravidez na adolescência para adolescentes grávidas. Rev Rene, 12(2). http://www.periodicos.ufc.br/rene/article/view/4226 
Bocardi, M. I. B., \& Cano, M. A. T. (1998). Gravidez na adolescência: o parto enquanto espaço do medo. In Livro de Resumos. EERP.

Brasil. (2000). Programa de Humanização no Pré-natal e nascimento. In Portaria $N^{\circ}$ 569/2000. Ministério da Saúde. https://bvsms.saude.gov.br/b vs/saudelegis/gm/2000/prt0569_01_06_2000_rep.html

Brasil. (2021). Sistema de Informações sobre Nascidos Vivos (SINASC) - Dados Abertos - Centrais de Conteúdos - DASNT - SVS/MS. Ministério Da Saúde, Coordenação-Geral de Informações e Análises Epidemiológicas. http://svs.aids.gov.br/dantps/centrais-de-conteudos/dados-abertos/sinasc/

Duarte, E. da S., Pamplona, T. Q., \& Rodrigues, A. L. (2018). A gravidez na adolescência e suas consequências biopsicossociais. . DêCiência Em Foco, 2(1), 45-52. http://revistas.uninorteac.com.br/index.php/DeCienciaemFoco0/article/view/145

Glêdes, M., Gurgel, I., Dalva, M., Alves, S., Rejane, E., Moura, F., Neyva Da Costa Pinheiro, P., Maria, R., \& Rego, V. (2010). Desenvolvimento De Habilidades: estratégia de promoção da saúde e prevenção da gravidez na adolescência Title: Skills development: strategy for health promotion and prevention of pregnancy in adolescence. Rev Gaúcha Enferm, 31(4), 640-646.

Lansky, S., Friche, A. A. de L., Silva, A. A. M. da, Campos, D., Bittencourt, S. D. de A., Carvalho, M. L. de, Frias, P. G. de, Cavalcante, R. S., \& Cunha, A. J. L. A. da. (2014). Pesquisa Nascer no Brasil: perfil da mortalidade neonatal e avaliação da assistência à gestante e ao recém-nascido. Cadernos de Saúde Pública, 30(SUPPL1), S192-S207. https://doi.org/10.1590/0102-311X00133213

Lima, E. F. de O., Miura, P. O., Santos. Kedma Augusto Martiniano, Galdino, E. B. T., \& Pedrosa, M. M. M. P. (2017). Gravidez na adolescência para os jovens: revisão sistemática. V Seminário Internacional Enlaçando Sexualidade. https://webcache.googleusercontent.com/search?q=cache:Mh4bSSQ2pigJ :htt ps://www.editorarealize.com.br/editora/anais/enlacando/2017/TRABALHO_EV072_MD1_SA17_ID1090_01082017172604.pdf+\&cd=1\&hl=pt-BR\&ct =cl nk\&gl=br

Minas Gerais. (2006). Atenção à saúde do adolescente: . In Secretaria de Estado de Saúde. (p. 152). SAS/MG. www.saude.mg.gov.br

Nasio, J.-D., \& tradução André Telles. (2011). Como agir com um adolescente difícil?: um livro para pais e profissionais. Zahar.

Oliveira, E. F. V. de, Gama, S. G. N. da, \& Silva, C. M. F. P. da. (2010). Gravidez na adolescência e outros fatores de risco para mortalidade fetal e infantil no Município do Rio de Janeiro, Brasil. Cadernos de Saúde Pública, 26(3), 567-578. https://doi.org/10.1590/S0102-311X2010000300014

Oliveira, P. R. de, Rodrigues, J. Z., Ferreira, J. D., Batista, D. J. R., Gusmão, R. M. de, Franco, S. E. de J., Rocha, E. M. da, \& Lemes, A. G. (2018). Gravidez na adolescência: um desafio crítico para os países do cone sul. Journal Health NPEPS, 3(2), 506-526. https://doi.org/10.30681/25261010

Oliveira, J. C., Silva, S. C. P., Ribeiro, V. D., Filipini, S. M., \& Leite, F. S. (2006). Conhecimento das gestantes sobre tipos de parto. In Universidade do Vale do Paraíba. (Ed.), X Encontro Latino Americano de Iniciação Científica e VI Encontro Latino Americano de Pós-Graduação.

Oliveira, L. L., Gonçalves, A. de C., Costa, J. S. D. da, \& Bonilha, A. L. de L. (2016). Fatores maternos e neonatais relacionados à prematuridade. Revista Da Escola de Enfermagem Da USP, 50(3). https://www.scielo.br/scielo.php?pid=S0080-62342016000300382\&script=sci_arttext\&tlng=pt

Organización Panamericana de la Salud, \& Organización Mundial de la Salud. (2016). Accelerating progress toward the reduction of adolescent pregnancy in Latin America and the Caribbean. Report of a technical consultation. https://www.unicef.org/lac/media/1336/file/PDF_Acelerar_el_progreso_h acia_la_reducción_del_embarazo_en_la_adolescen.pdf

Piccinini, C. A., Da Rosa, M., Tonantzin, S., Gonçalves, R., Lopes, R. S., \& Tudge, J. (2044). O Envolvimento Paterno durante a Gestação. Psicologia: Reflexão e Crítica, 17(3), 303-314. psicologia: Reflexão e Crítica, 2004, 17(3), pp.303-314

Ribeiro, V., Ribeiro, V. C. da S., Nogueira, D. L., Assunção, R. S., Silva, F. M. de R. e, \& Quadros, K. A. N. (2016). Papel do enfermeiro da estratégia de saúde da família na prevenção da gravidez na adolescência. Revista de Enfermagem Do Centro-Oeste Mineiro, O(0). https://doi.org/10.19175/recom.v0i0.881

Santos, D. R. dos, Maraschin, M. S., \& Caldeira, S. (2008). Percepção dos enfermeiros frente à gravidez na adolescência. Ciência, Cuidado e Saúde, 6(4), 479-485. https://doi.org/10.4025/cienccuidsaude.v6i4.3684

Schwanke, M., \& Pinto, A. B. (2011). A percepção dos adolescentes residentes no município de Alto Bela Vista - SC sobre gravidez na adolescência. AGORA: Revista de Divulgação Científica, 16(2(A)). http://webcache.googleusercontent.com/search?q=cache:WbFXnImQzWcJ:www.peri odicos.unc.br/index.php/agora/article/viewFile/106/184+\&cd=2\&hl=pt-BR\&ct=clnk\&gl=br

Sociedade Brasileira de Pediatria. (2019, January). Prevenção da Gravidez na Adolescência. Guia Prático de Atualização.

Suzuki, C. M., Esther, M., Ceccon, J., Falcão, M. C., \& Adolfo Costa Vaz, F. (2007). Análise comparativa da frequência de prematuridade e baixo peso entre filhos de mães adolescentes e adultas. Rev Bras Crescimento Desenvolv Hum, 17(3), 95-103.

Valentim, T. G. S. (2018). Impacto da gravidez na adolescência nos resultados perinatais: uma revisão integrativa. In Monografia - Universidade Federal do Maranhão (p. 58).

WHO. (2019). ICD-11: International Classification of Diseases . World Health Organization. https://icd.who.int/en 